\title{
Induction of remifentanil by bradycardia and hypotension through distinct biochemical mechanisms
}

\author{
Yong Yang ${ }^{1}$, Qu-Lian Guo ${ }^{1}$, Di Fu ${ }^{1}$, Hui Wu ${ }^{3}$ and Ru-Ping Dai ${ }^{2 *}$ \\ ${ }^{1}$ Department of Anaesthesia, Xiang-Ya Hospital, Central South University, Changsha, Hunan 410011, China. \\ ${ }^{2}$ Department of Anaesthesia, The Second Xiangya Hospital of Central South University, Changsha, \\ Hunan 410011, China. \\ ${ }^{3}$ Department of Medical Oncology, Hunan Provincial Tumor Hospital, Changsha, China.
}

Accepted 5 July, 2012

\begin{abstract}
Remifentanil has been well known to cause much more significant bradycardia and hypotension as compared with other opioids. However, the underlying mechanism is still poorly understood. The present study hypothesized that remifentanil led to bradycardia and hypotension with distinct mechanisms and aimed to test this hypothesis. Testing the hypothesis would broaden our understanding the underlying mechanism of remifentanil and may provide some guidance in the use of remifentanil clinically. Mean arterial pressure and heart rate were measured before and at the indicated time after drug treatment in the anesthetized rabbits. In the vagotomy group, bilateral vagus in the neck was exposed and one section of the vagus severed respectively. Remifentanil caused slight but significant bradycardia immediately which recovered entirely within one minute. On the other hand, bolus injection of remifentanil led to severe hypotension which sustained for more than five minutes. Bilateral vagotomy could delay the recovery of remifentanil-mediated bradycardia but did not affect remifentanil-evoked hypotension significantly. Pretreatment with naloxone, a non-specific opioid receptor antagonist could entirely prevent the remifentanil-mediated hypotension but only partially reversed remifentanil-mediated bradycardia. Remifentanil induces bradycardia and hypotension with distinct physiological and biochemical mechanisms. Remifentanil-induced bradycardia is mediated by opioid receptor-dependent and -independent pathways; however, remifentanil-induced hypotension is only through opioid receptor-dependent pathway.
\end{abstract}

Key words: Remifentanil, bradycardia, hypotension, opioid receptor, hemodynamic effect.

\section{INTRODUCTION}

Remifentanil is a potent opioid of the 4-anilidopiperidine type with a similar pharmacodynamic property to other opioids. The major difference in chemical structure of remifentanil with other opioids is that remifentanil contains methyl ester at $\mathrm{N}$-acyl moiety which can be cleaved by widespread non-specific esterases, present ubiquitously in the tissue and plasma. Thus, as compared with other opioids, remfentanil has a unique pharmacokinetic characteristic with a very short half time and therefore widely used in clinical anesthesia (Beers

\footnotetext{
*Corresponding author. E-mail: Ruping_dai@yahoo.com.cn.
}

and Camporesi, 2004). However, it has been widely known that remifentanil has more severe hemodynamic consequences compared with other opioids (Elliott et al., 2000; Kazmaier et al., 2000; Wang et al., 1999). In spite of multiple mechanisms proposed including central vagal activation (Garofalo et al., 2008), direct negative chronotropic effect (Zaballos et al., 2009), peripheral vasodilation (Unlugenc et al., 2003), there is still not a consistent conclusion yet. For example, using echocardiography, a previous study suggested that the decreased blood pressure was caused by the reduction of heart rate (HR) (Chanavaz et al., 2005). However, another study showed that remifentanil decreased mean arterial pressure (MAP) in the patients with artificial heart 
in which the HR was fixed (Ouattara et al., 2004). In addition, it has also been reported that remifentanil could lead to the vasodilation through the endothelium-released nitric oxide in the in vitro aorta and remifentanil could decrease systemic vascular resistance thus resulting in the hypotension (Unlugenc et al., 2003). These findings suggest that remifentanil induces bradycardia and hypotension through distinct physiological mechanisms. In addition, remifentanil has more robust depressed cardiovascular effect than other $\mu$ opioid agonists in similar analgesia doses. This suggests that remifentanil may exert its cardiovascular effect through opioid receptor independent pathways. The present study thus hypothesized that remifentanil induced bradycardia and hypotension through distinct mechanisms and aimed to test this hypothesis in the rabbit model. Testing the hypothesis would broaden our understanding the underlying mechanism of remifentanil and may provide some guidance in the use of remifentanil clinically.

\section{MATERIALS AND METHODS}

\section{Animals}

The study was carried out on male New Zealand white rabbits (2.5 to $3.5 \mathrm{~kg}$ ) obtained from Central South University Animal Services (Changsha, China). A total of 64 rabbits were used in the different experimental protocols. In each protocol, eight rabbits were used for each group. The experimental protocol was approved by the Animal Care and Use Committee of Central South University and conformed to the National Institutes of Health Guide for the Care and Use of Laboratory Animals. All efforts were made to minimize the number of rabbits used and their suffering.

\section{Preparation}

Animals were placed with polythylene catheters in the ear vein for administration of drugs and saline. Anesthesia was induced by 25 $\mathrm{mg} / \mathrm{kg}$ pentobarbital sodium and maintained by $10 \mathrm{mg} / \mathrm{kg} / \mathrm{h}$ continuous infusion. Animals were paralyzed with $0.1 \mathrm{mg} / \mathrm{kg}$ vecuronium and infused continuously with $10 \mathrm{ml} / \mathrm{kg} / \mathrm{h}$ Ringer's lactate solution to maintain the blood volume throughout the experiments. The animals were then tracheotomized and the lungs were ventilated with a small animal ventilator (Harvard Medical Apparatus, USA). Polythylene catheters were placed in internal jungular artery for measurement of arterial pressure with a pressure transducer and sampling of arterial blood. HR was recorded from lead II of the electrocardiogram (Model, HP). Acid-base balance was maintained within normal limits $\left(\mathrm{PaCO}_{2}, 35\right.$ to $45 \mathrm{mmHg}, \mathrm{pH}$ 7.35 to 7.45 ) by adjusting the tidal volume and frequency. The $\mathrm{PaO}_{2}$ was maintained between 100 and $200 \mathrm{mmHg}$. Bilateral cervical vagotomy was performed by isolating and severing bilateral vagus nerve from the vagina carotica at the first tracheal ring (Farber, 1937).

\section{Experimental protocol}

The study firstly compared the hemodynamic response of different doses of remifentanil (Renfu pharmaceutics, Hubei, China) with similar analgesic dose of fentanyl. Three different doses of remifentanil $(1.0,2.5$ and $5 \mu \mathrm{g} / \mathrm{kg}$ ) and $5 \mu \mathrm{g} / \mathrm{kg}$ fentanyl rapidly were administered to investigate the hemodynamic response. $\mathrm{HR}$ and MAP were recorded at $30 \mathrm{~s}, 1,2,3,5$ and 10 or 20 min after drug injection.

\section{Experimental protocol II}

The present study also aimed to investigate the role of vagus in the hemodynamic response of remifentanil. Animals were divided randomly into two groups, group I: remifentanil only group (REM) and group II: vagotomy group (REM + Vag). $5 \mu \mathrm{g} / \mathrm{kg}$ remifentanil was administered to the different groups and HR and MAP were recorded in the indicated times after drug administration.

\section{Experimental protocol III}

To investigate whether REM-induced hypotension and bradycardia was opioid receptor dependent, naloxone was injected before administration of remifentanil. After $2 \mathrm{~min}, 5 \mu \mathrm{g} / \mathrm{kg}$ remifentanil was administered, and HR and MAP were recorded at different times after REM administration.

\section{Statistical analysis}

Statistical analysis was performed using software Prism 5.0 (Graphpad Software, San Diego, CA). Data are given as mean (95\% confidence interval, Cl). Differences between groups were compared using repeated measure analysis of variance (ANOVA) followed by post hoc Dunnett testing, Tukey testing or Bonferroni testing where appropriate. A value of $P<0.05$ was considered as significant difference.

\section{RESULTS}

There is no significant difference among age and weight in different experimental groups of animals. No significant difference was observed among the basal HR and MAP in all groups of animals. The mean of baseline HR in all groups of animals is 265 per min [95\% confidence interval (Cl), 250 to 280.6]. Mean of baseline MAP is $87.31 \mathrm{mmHg}(\mathrm{Cl}, 82.6$ to $92.03 \mathrm{mmHg})$.

\section{Dose-dependent effect of remifentanil on HR and MAP}

Three different doses of remifentanil $(1,2.5$ and $5 \mu \mathrm{g} / \mathrm{kg})$ and $5 \mu \mathrm{g} / \mathrm{kg}$ fentanyl were administered to compare the cardiovascular effect of remifentanil with fentanyl. As shown in Figure 1A, $5 \mu \mathrm{g} / \mathrm{kg}$ fentanyl only slightly decreases HR with $9.54 \%(\mathrm{Cl}, 6.45 \%$ to $11.25 \%)$ at $30 \mathrm{~s}$ after administration of drugs $(p=0.008, n=8)$. After that, the decreased HR recovers and returns to the baseline level ( $p>0.05$ vs baseline). Similarly, 1 and $2.5 \mu \mathrm{g} / \mathrm{kg}$ remifentanil also decreases $\mathrm{HR}$ around $8.19 \%(\mathrm{Cl}, 7.23$ to $11.2 \%)$ and $11.19 \%(\mathrm{Cl}, 9.85$ to $12.76 \%)$ respectively at $30 \mathrm{~s}$ after drug administration. The reduced HR returns to the baseline at $1 \mathrm{~min}$ and later time points. Bolus injection of $5 \mu \mathrm{g} / \mathrm{kg}$ remifentanil reduces $\mathrm{HR}$ with $15.47 \%$ 

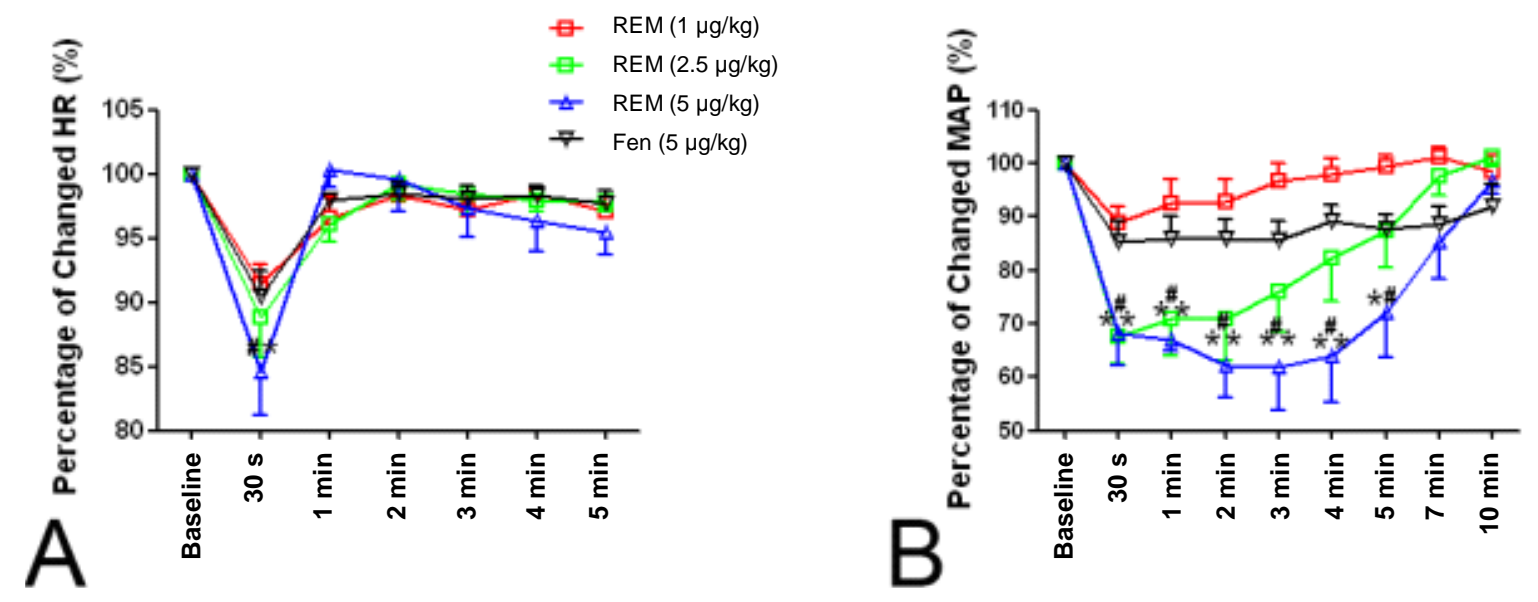

Figure 1. Time course changes (\%) of HR (A) and MAP (B) after bolus injection of different doses (1, 2.5 and 5 $\mu \mathrm{g} / \mathrm{kg}$ ) of remifentanil and $5 \mu \mathrm{g} / \mathrm{kg}$ fentanyl. REM, remifentanil; Fen, Fentanyl Values are mean \pm SEM. ${ }^{*}, \mathrm{p}<0.05$ vs Baseline, ${ }^{* *}, p<0.01$ vs Baseline, $\#, p<0.05$ vs fentanyl group, $n=8$ for each group.
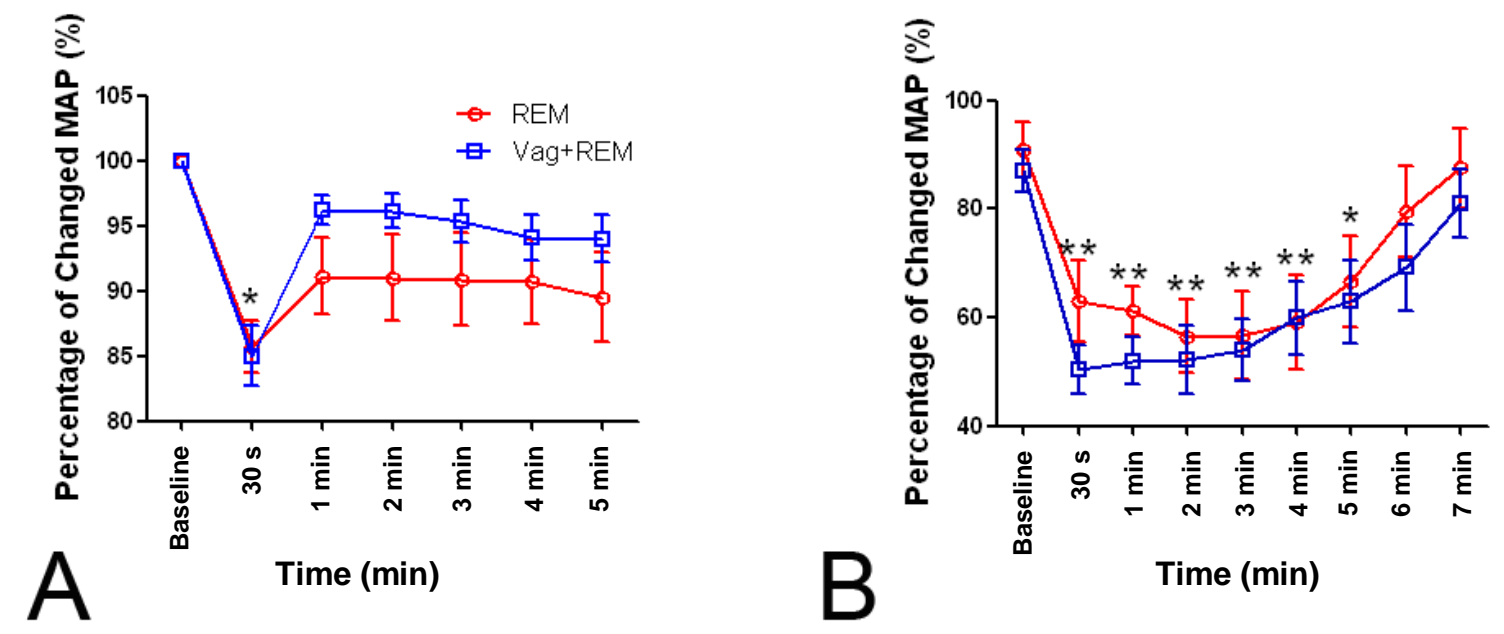

Figure 2. Effect of bilateral vagotomy (REM + Vag) on the decreased HR $(A)$ and MAP $(B)$ after bolus injection of 5 $\mu \mathrm{g} / \mathrm{kg}$ remifentanil. Values are mean \pm SEM. ${ }^{*}, p<0.05$ different experimental groups vs baseline; ${ }^{* *}, p<0.01$ different groups vs baseline. $\mathrm{N}=8$ for each group.

(CI, 13.24 to $18.35 \%)$ immediately as compared to baseline. Repeated measures ANOVA followed by Tukey test analysis shows that the $5 \mu \mathrm{g} / \mathrm{kg}$ remifentanil produces more severe bradycardia than $5 \mu \mathrm{g} / \mathrm{kg}$ fentanyl $\left(\mathrm{t}_{2,14}=2.73, \mathrm{p}=0.025\right)$ and $1 \mu \mathrm{g} / \mathrm{kg}$ remifentanil $\left(\mathrm{t}_{2,14}=\right.$ $3.18, p=0.012)$. These data suggest that remifentanil rapidly but transiently reduces $\mathrm{HR}$ in a dose-dependent manner.

As shown in Figure 1B, fentanyl $(5 \mu \mathrm{g} / \mathrm{kg})$ slightly reduces the MAP after drug administration $\left(\mathrm{F}_{8,73}=5.73, \mathrm{p}\right.$ $=0.0008)$. However, 2.5 and $5 \mu \mathrm{g} / \mathrm{kg}$ remifentanil dramatically reduces the MAP by more than $30 \%(\mathrm{Cl}$, 32.3 to $37.6 \%)$ at $30 \mathrm{~s}$ after drug administration $\left(\mathrm{F}_{8,71}=\right.$ $5.818, p=0.00009$ ). The decreased MAP responding to 5 $\mu \mathrm{g} / \mathrm{kg}$ remifentanil sustains for more than $5 \mathrm{~min}$. At $5 \mathrm{~min}$ after $5 \mu \mathrm{g} / \mathrm{kg}$ administration, the MAP is still only around
$72 \%(\mathrm{Cl}, 65.7$ to $75.3 \%)$ of MAP at the basal level $(p<$ 0.001 , One way ANOVA followed by Dunnet test).

\section{Effect of vagotomy on remifentanil-induced hypotension and bradycardia}

In an attempt to examine whether vagal activation is involved in the hypotension and bradycardia by remifentanil, bilateral cervical vagotomy was performed. After $30 \mathrm{~min}$ for stabilization, the effects of $5 \mu \mathrm{g} / \mathrm{kg}$ remifentanil on HR and MAP were examined. As shown in Figure 2, in the group with bilateral vagotomy, remifentanil-induced bradycardia gradually returned to the baseline (Figure 2A and B). There was no significant difference of changes of MAP after vagotomy (Figure $2 \mathrm{~A}$ and $\mathrm{B})(\mathrm{F} 3,24=0.24, \mathrm{p}=0.867$, repeated measures. 

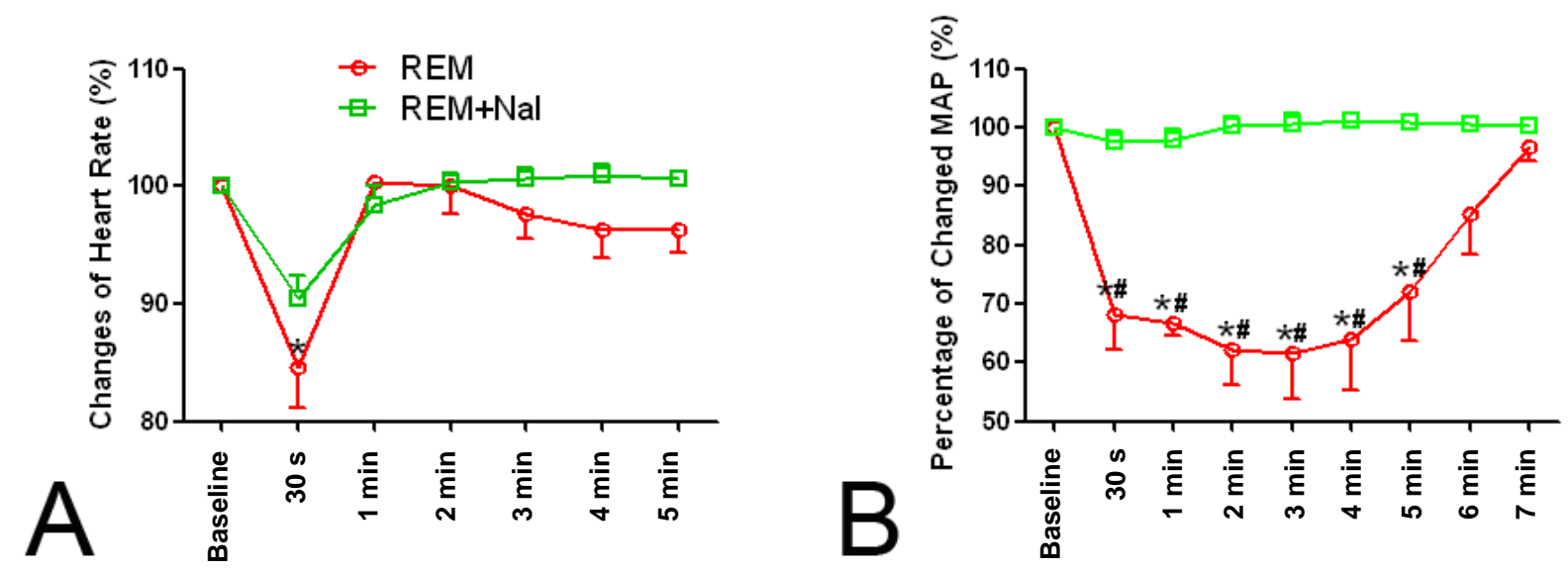

Figure 3. Effect of naloxone $(40 \mu \mathrm{g})$ on the decreased HR (A) and MAP (B) responding to remifentanil $(5 \mu \mathrm{g} / \mathrm{kg}) \mathrm{bolus}$ injection. Values are mean \pm SEM. Naloxone totally prevented the decreased MAP (B) by remifentanil administration but only partially attenuated the decreased HR (A). REM, remifentanil *, $p<0.05$ vs baseline; ${ }^{*}, p<0.05$ vs naloxone pretreatment group. $\mathrm{N}=8$ for each group.

\section{Effect of naloxone on remifentanil-induced decreased HR and MAP}

To determine whether remifentanil-induced hypotension and bradycardia was opioid receptor dependent, naloxone $(40 \mu \mathrm{g})$ was administered $2 \mathrm{~min}$ before remifentanil was injected. As shown in Figure 3 , in the groups with naloxone pretreatment, HR at $30 \mathrm{~s}$ after remifentanil $(5 \mu \mathrm{g} / \mathrm{kg})$ injection was $8.86 \%$ less than the basal level ( $t=9.704, p=0.0006$ vs baseline, repeated measures one way ANOVA followed by Tukey test) (Figure 3A). However, in the group with naloxone pretreatment, MAP is not significantly changed at all time measured after remifentanil bolus injection $\left(F_{8,56}=1.153\right.$, $p=0.34$, repeated measures one way ANOVA followed by Tukey test) (Figure 3B). These findings suggest that naloxone completely prevents remifentanil-evoked hypotension but only partially inhibits bradycardia.

\section{DISCUSSION}

There are several interesting findings in the present study. First, remifentanil-evoked bradycardia and hypotension are dissociated. In this regard, remifentanilevoked hypotension was more potent and sustained longer than remifentanil-induced bradycardia. Second, vagus denervation by bilateral vagotomy did not prevent the hemodynamic changes in response to remifentanil administration. Third, naloxone pretreatment completely prevented the decreased MAP whereas it only partially prevented bradycardia induced by remifentanil.

In a similar model, a previous study reported that the maximum of decrease in HR in response to remifentanil was approximately $15 \%$ and recovered within $1 \mathrm{~min}$ (Shinohara et al., 2000). In agreement with this, the present study also showed that remifentanil decreased HR mildly and shortly. However, inconsistent with the earlier study in which the time course of hypotension responding to remifentanil was correlated with that of $\mathrm{HR}$, remifentanil-induced hypotension sustained more than 5 min when HR had been entirely recovered already. The discrepant response of blood pressure between the present study and earlier study may be due to different anesthetics or experimental manipulations. Notably, in this study, the anesthetic was barbiturates whereas urethane was used in the earlier study. In addition, in the present study, remifentanil was injected faster in the present study than the earlier study. Despite this discrepancy, the present study suggested that there is temporal dissociation between hypotension and bradycardia in response to remifentanil. In addition, in the present study, remifentanil-induced hypotension was much more dramatically ( $40 \%$ of baseline) higher than bradycardia $(\sim 15 \%$ of baseline). All these findings suggest that remifentanil-induced hypotension and HR are due to different mechanisms. Though, a unifying hypothesis is preferred to explain the cardiovascular response of remifentanil the results obain indicate otherwise. Supporting this assumption, a recent clinical study also showed that atropine could prevent the decreased HR but not the fall of MAP (Maruyama et al., 2010).

Several physiological mechanisms were proposed to interpret remifentanil-induced bradycardia including the central vagotomic activation, the negative chronotropic effect and direct effect on cardiac conduction (sinus node and atriaventricular node) (Fujii et al., 2011; Zaballos et al., 2009). In the present study, bilateral vagotomy did not reverse remifentanil-induced bradycardia suggesting that central vagotomy may not contribute to the bradycardia induced by remifentanil. This finding is consistent with a 
clinical study which reported that the negative chronotropic effect, but not increased parasympathetic activity, contributed to remifentanil-induced bradycardia (Tirel et al., 2005) and supports the assumption that other effect of remifentanil such as direct effect of conduction system regulated remifentanil-evoked bradycardia. On the other hand, many studies have clearly demonstrated that remifentanil has direct vasodilator effect. For example, in the cultured human radial artery, remifentanil was shown to induce vasorelaxation in a concentrationdependent manner (Gursoy et al., 2006). In another clinical study, remifentanil still decreased MAP in the patients with artificial heart suggesting the direct effect on blood vessels but not mediated by the reduction of HR (Ouattara et al., 2004). In the present study, bilateral vagotomy did not attenuate the hypotension induced by remifentanil suggesting that the direct vasodilator effect by remifentanil contributes to the hypotension after remifentanil treatment.

The most interesting observation in the present study was that naloxone totally reversed hypotension but only marginally attenuated bradycardia after remifentanil administration. These data strongly indicate that remifentanil induces bradycardia and hypotension through distinct biochemical pathways. Surprisingly, the effect of naloxone on remifentanil-induced hypotension or bradycardia has never been tested in the intact animals or human beings despite the report in the barodenervation rabbits. The fact that remifentanil-induced hypotension was entirely prevented by non specific opioid receptor antagonist naloxone implies that remifentanilevoked hypotension was through opioid receptordependent pathway. However, naloxone only partially prevented bradycardia indicating that REM-induced bradycardia is also mediated by opioid receptorindependent pathway. Despite multiple physiological mechanisms, it seems undoubting that remifentanil exerts its pharmacological effect through opioid receptor from biochemical insights. This is because remifentanil is designed to possess an ester linkage which allows it metabolized by plasma and tissue non-specific esterases. Thus, the supposed key difference between remifentanil and fentanyl is the pharmacokinetics but not its molecular targets. However, after adding an ester linkage, remifentanil may also act on other receptors which cannot be inhibited by naloxone. The existence of unknown downstream signaling of remifentanil may also explain why remifentanil produces more potent bradycardia and hypotension than fentanyl. On the other hand, given that $\mu$ opioid receptor is widely distributed in the endothelium of the blood vessels, remifentanil administration results in hypotension through acting on opioid receptor and dilating the blood vessels subsequently. Taken together, these findings suggest that opioid receptor-dependent pathway is responsible for remifentanil-induced hypotension but only partially contributes to bradycardia.
One limitation should be considered before assessing the clinical significance of the current study. The administration dose $(5 \mu \mathrm{g} / \mathrm{kg})$ was higher than that used in clinical practice. In addition, injection speed was faster than that in clinical anesthesia. Thus, the experimental protocol may not be applied in the clinical practice. However, in clinical anesthesia, lower dose of remifentanil may also exert more persistent and robust hypotension than bradycardia when used together with other anesthetics such as propofol or midazolam. Thus, other vasoactive drugs may be needed to prevent hypotension clinically.

In conclusion, the findings of the study suggest that remifentanil mediates bradycardia and hypotension through distinct physiological and biochemical mechanisms. Physiologically, central vagal activity may be involved in the induction of bradycardia and hypotension by remifentanil but not contribute to the later phase of hypotension. On the other hand, remifentanil-induced hypotension is mediated by opioid receptor-dependent pathway whereas bradycardia is mediated by opioid receptor-dependent and -independent pathways. The distinct biochemical mechanism may be able to explain the more potent cardiovascular consequence of remifentanil than other opioids. However, the findings in the animal study may be needed to be confirmed in the clinical study in the future study.

\section{ACKNOWLEDGEMENT}

This study is supported by National Natural Science Foundation of China, Beijing, China (Grant No: 81070897).

\section{REFERENCES}

Beers R, Camporesi E (2004). Remifentanil update: clinical sci. and utility. CNS. Drugs 18:1085-1104.

Chanavaz C, Tirel O, Wodey E, Bansard JY, Senhadji L, Robert JC, Ecoffey C (2005). Haemodynamic effects of remifentanil in children with and without intravenous atropine. An echocardiographic study. Br. J. Anaesth. 94:74-79.

Elliott P, O'Hare R, Bill KM, Phillips AS, Gibson FM, Mirakhur RK (2000). Severe cardiovascular depression with remifentanil. Anesth. Analg. 91:58-61.

Farber S (1937). Studies of pulmonary edema. I. The consequences of bilateral cervical vagotomy in rabbit. J. Exp. Med. 66:397-404.

Fujii K, Iranami H, Nakamura Y, Hatano Y (2011). High-dose remifentanil suppresses sinoatrial conduction and sinus node automaticity in pediatric patients under propofol-based anesthesia. Anesth. Analg. 112:1169-1173.

Garofalo NA., Teixeira-Neto FJ, Schwartz DS, Vailati Mdo C, Steagall PV (2008). Effects of the opioid remifentanil on the arrhythmogenicity of epinephrine in halothane-anesthetized dogs. Can. J. Vet. Res. 72:362-366.

Gursoy S, Bagcivan I, Yildirim MK, Berkan O, Kaya T (2006) Vasorelaxant effect of opioid analgesics on the isolated human radial artery. Eur. J. Anaesthesiol. 23:496-500.

Kazmaier S, Hanekop GG, Buhre W, Weyland A, Busch T, Radke OC, Zoelffel R, Sonntag H (2000). Myocardial consequences of remifentanil in patients with coronary artery disease. Br. J. Anaesth. 
84:578-583.

Maruyama K, Nishikawa Y, Nakagawa H, Ariyama J, Kitamura A, Hayashida M (2010). Can intravenous atropine prevent bradycardia and hypotension during induction of total intravenous anesthesia with propofol. remifentanil? J. Anesth. 24:293-296.

Ouattara A, Boccara G, Kockler U, Lecomte P, Leprince P, Leger P, Riou $B$, Rama A, Coriat $P$ (2004). Remifentanil induces systemic arterial vasodilation in humans with a total artificial heart. Anesthesiol. 100:602-607.

Shinohara K, Aono H, Unruh GK, Kindscher JD, Goto H (2000). Suppressive effects of remifentanil on hemodynamics in barodenervated rabbits. Can. J. Anaesth. 47:361-366.

Tirel O, Chanavaz C, Bansard JY, Carre F, Ecoffey C, Senhadji L, Wodey E (2005). Effect of remifentanil with and without atropine on heart rate variability and RR interval in children. Anaesthesia 60:982989.
Unlugenc H, Itegin M, Ocal I, Ozalevli M, Guler T, Isik G (2003). Remifentanil produces vasorelaxation in isolated rat thoracic aorta strips. Acta Anaesthesiol. Scand. 47:65-69.

Wang JY, Winship SM, Thomas SD, Gin T, Russell GN (1999). Induction of anaesthesia in patients with coronary artery disease: a comparison between sevoflurane-remifentanil and fentanyl-etomidate. Anaesth. Intensive Care 27:363-368.

Zaballos M, Jimeno C, Almendral J, Atienza F, Patino D, Valdes E, Navia J, Anadon MJ (2009). Cardiac electrophysiological effects of remifentanil: study in a closed-chest porcine model. Br. J. Anaesth. 103:191-198 